\title{
Noise-enhanced excitability in bistable activator-inhibitor media
}

\author{
J. García-Ojalvo* \\ Departament de Física i Enginyeria Nuclear, Universitat Politècnica de Catalunya, Colom 11, E-08222 Terrassa, Spain
}

F. Sagués

Departament de Química Física, Universitat de Barcelona, Diagonal 647, E-08028 Barcelona, Spain

J. M. Sancho

Departament d'Estructura i Constituents de la Matèria, Universitat de Barcelona, Diagonal 647, E-08028 Barcelona, Spain

L. Schimansky-Geier

Institut für Physik, Humboldt-Universität zu Berlin, Invalidenstrasse 110, D-10115 Berlin, Germany

(Received 3 September 2001; published 17 December 2001)

\begin{abstract}
We show that external fluctuations induce excitable behavior in a bistable spatially extended system with activator-inhibitor dynamics of the FitzHugh-Nagumo type. This can be understood as a mechanism for sustained signal propagation in bistable media. The phase diagram of the stochastic system is analytically obtained and numerically verified. For small-noise intensities, front propagation becomes unstable, and excitable pulses arise as the only possible spatiotemporal behavior of the system. For large-noise intensities, on the other hand, the system enters an effective regime of oscillatory behavior, where it exhibits spontaneous nucleation of pulses and synchronized firing.
\end{abstract}

\section{DOI: 10.1103/PhysRevE.65.011105}

\section{INTRODUCTION}

Excitability is one of the most important mechanisms of sustained signal transmission in nature. Excitable systems have only one stable steady state, but respond to weak perturbations in a nontrivial way, performing a large excursion in phase space before eventually returning to their original rest state. Such behavior provides these systems with a selfresetting mechanism after excitation which, complemented with spatial diffusion, allows for the self-sustained propagation of waves, in the form of pulses in one-dimensional channels and spiral waves (among other structures) in twodimensional media [1]. These phenomena are of utmost importance, in particular, in physiology, where they constitute the main mechanism by which signals propagate through nerve and cardiac cells, for instance [2]. In the specific case of neural tissue, propagation takes place in a substantially noisy environment, and thus it seems natural to examine what is the role of random fluctuations in this dynamical behavior. In this direction, previous studies have shown that noise is able to produce collective bursts in a simple model of the visual cortex [3], induce spiral-wave propagation [4] and pacemaker activity $[5,6]$ in excitable cellular automata, drive the emission of circular and spiral waves in cellular slime molds [7], generate calcium waves in glial-cell networks [8], and give rise to synchronized firing in cellular automaton models of excitable media [9] and in models of coupled neurons $[10,11]$. In this latter situation, random excitations become entrained to the characteristic time scales of the deterministic dynamics for an optimal amount of noise, locally inducing an "autonomous" coherent response of the system [12], and spatial coupling synchronizes the resulting

\footnotetext{
*Electronic address: jordi.g.ojalvo@upc.es
}

PACS number(s): 05.40.-a, 87.10.+e

local coherent behavior among the different oscillators [13].

In all the above-mentioned situations, the underlying deterministic state of the system is originally excitable, and noise only produces a certain spatiotemporal behavior in the medium. A more fundamental question is whether noise is able to induce the excitable behavior itself. The answer is again positive, as shown experimentally in a subexcitable chemical medium undergoing a photosensitive BelousovZhabotinsky reaction, which can be rendered excitable by an externally added noise in the illumination profile [14]. Recent experiments in the same system have also shown noiseinduced transitions from an excitable to an oscillatory regime [15]. In this paper, we address the question of whether excitable behavior can also be induced in bistable media.

In general, bistable systems are not suitable media for signal-transmission purposes, since under normal conditions they only support propagation of fronts, and therefore a certain external resetting mechanism is required for the transmission of information-carrying pulses. In any case, noise has already proven helpful to sustain propagation of fronts in chains of bistable diode oscillators [16] and of harmonic signals in coupled double-well potential models [17]. Additionally, an artificial model of a one-component bistable medium has also been developed that exhibits pulse propagation sustained by noise [18]. In what follows, we show that external random fluctuations naturally sustain propagation of pulses in standard models of activator-inhibitor media operating in a deterministically bistable regime, where only propagation of fronts would take place in the absence of noise under simple initial conditions (e.g., as a response to a local perturbation of a homogeneous steady state). This is accomplished by means of a noise-induced transition from the bistable to an excitable regime, as advanced in the previous paragraph.

Activator-inhibitor media are usually modeled by means of two coupled reaction-diffusion equations, a particularly 
important example of which is given by the well-known FitzHugh-Nagumo class of models, whose general form is

$$
\begin{gathered}
\frac{\partial u}{\partial t}=\frac{1}{\varepsilon} f(u, v)+\frac{\partial^{2} u}{\partial x^{2}}, \\
\frac{\partial v}{\partial t}=g(u, v),
\end{gathered}
$$

where $u(x, t)$ represents the activator and $u(x, t)$ the inhibitor, and $\varepsilon \ll 1$, so that the activator evolves in a time scale much faster than the inhibitor (whose diffusion is also neglected). For specific choices of the reaction terms $f(u, v)$ and $g(u, v)$, this model constitutes a very useful simplified description of electrical signal transmission along neuron axons, with $u$ representing the membrane potential and $v$ being related to the time-dependent conductance of the potassium channels $[2,19]$. We aim to examine here the influence of an external multiplicative noise in the dynamics of the activator when the system is deterministically bistable. Previous works have analyzed the effect of a fluctuating inhibitor dynamics in the excitable regime, showing that an additive noise can lead to synchronized firing [11], whereas a multiplicative noise is able to induce spiral breakup and turbulence [20]. This last type of fluctuating dynamics has also been examined in the bistable regime, where excitablelike structures were found [21]. In that case, however, such structures were produced by noise-induced decays from the "excited" stable state, and not by a real noise-induced transition between the two regimes, as reported below.

In what follows, we examine the dynamics of a particular type of stochastic FitzHugh-Nagumo model, namely, the Barkley model with multiplicative noise, operating in a deterministically bistable regime. A moderate amount of noise will be seen to greatly enhance excitable behavior in the system, whereas for larger noise intensities, the system exhibits oscillatory dynamics leading to synchronized firing.

\section{THE BISTABLE BARKLEY MODEL}

The Barkley model is a computationally efficient version of the FitzHugh-Nagumo activator-inhibitor dynamics. It is defined by the following choices for the reaction terms $f(u, v)$ and $g(u, v)[22]$

$$
\begin{gathered}
f(u, v)=u(1-u)\left(u-\frac{v+b}{a}\right), \\
g(u, v)=\gamma u-v,
\end{gathered}
$$

where $a, b$, and $\gamma$ are constant parameters that determine the type of local dynamics exhibited by the system, which can vary among purely monostable, oscillatory, excitable, and bistable. In what follows, we will concentrate on the two latter situations, which are represented in Fig. 1 in terms of the phase plane $(u, v)$. In that figure, solid lines represent the activator nullclines, defined by $f(u, v)=0$, and dashed lines correspond to the inhibitor nullcline $g(u, v)=0$.
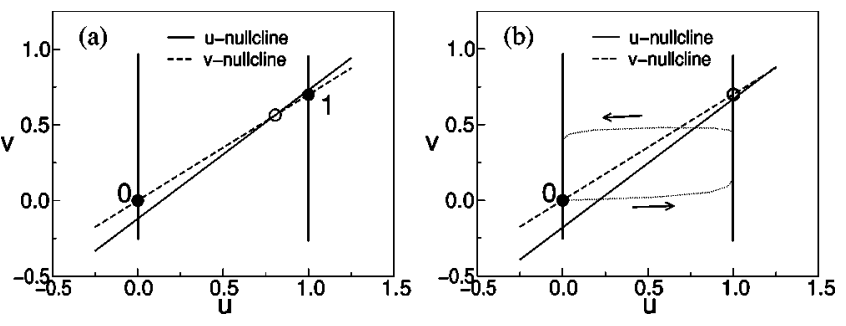

FIG. 1. Nullclines of model (2) for $a=0.85$ and $\gamma=0.7$, in the (a) bistable $[b=0.12]$, and (b) excitable $[b=0.18]$ regimes. Solid (empty) circles denote stable (unstable) fixed points.

For the parameter values chosen in Fig. 1(a), the two sets of nullclines intersect in three fixed points, two of which, labeled 0 and 1 in the figure, can be seen to be stable. This bistable behavior disappears for increasing $b$, when a transcritical bifurcation involving the stable steady-state 1 and the unstable fixed point appears, leading to the excitable regime represented in Fig. 1(b) [23]. In this situation, the system possesses a single stable steady state (rest state) [24], but a perturbation of sufficient (but small) intensity takes it to the excited branch at $u \approx 1$ [see dotted trajectory in Fig. 1(b)], where it follows the vertical $(u=1)$ nullcline upwards until it goes back again to the $(u=0)$ nullcline, and down to the rest state. This excitation plus resetting mechanism leads, in conjunction with spatial coupling, to the propagation of pulses through the medium.

We note that single pulse propagation can also occur in the bistable regime of Fig. 1(a), provided the initial conditions of the system are carefully prepared [25]. However, under general initial conditions, such as a single local perturbation in the activator profile, a front instead of a pulse will arise. This contrasts with the situation in the excitable regime, where fronts never exist, and pulses are the only possible spatiotemporal behavior. In order to make this difference clear, we now examine the response of the system to an instantaneous local perturbation of the activator profile. The spatiotemporal behavior of the activator variable $u$ corresponding to the two regimes represented in Fig. 1 is plotted in gray scale in Fig. 2. In the two cases, the system is initially in the rest state 0 everywhere, and only the value of the activator variable at the left boundary of the system is set above excitation threshold. For this simple (and realistic) initial condition, the system can only develop a front in the bistable regime [Fig. 2(a)], whereas it generates a welldefined pulse in the excitable regime [Fig. 2(b)]. In plot 2(a), boundary conditions are absorbing, whereas in plot 2(b), they are set to periodic as soon as the traveling pulse is developed, in order to trap the pulse in a ring, so that its long-term evolution can be systematically analyzed. The model is integrated on a one-dimensional (1D) lattice of 1000 cells with a spacing $\Delta x=0.25$, making use of a semiimplicit algorithm for the activator equation [26], and an explicit algorithm for the inhibitor one.

\section{BARKLEY MODEL WITH STOCHASTIC ACTIVATOR DYNAMICS}

We now assume that the control parameter $b$ is subjected to random fluctuations evolving at time scales much shorter 
(a)

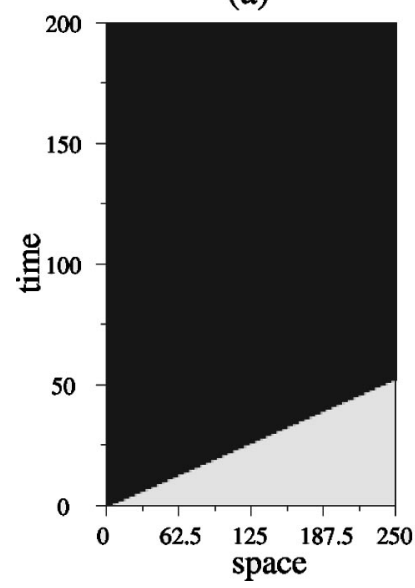

(b)

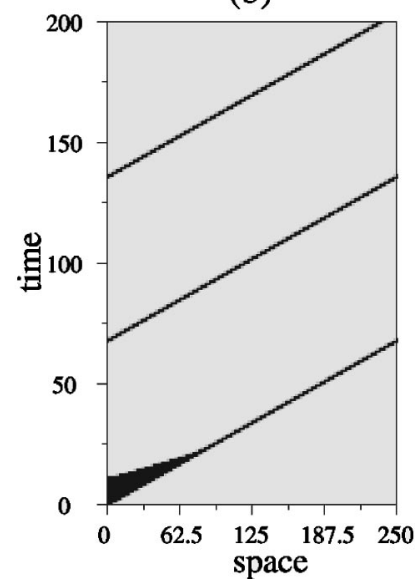

FIG. 2. Spatiotemporal evolution of model (2) corresponding to the two situations of Fig. 1: (a) bistable and (b) excitable. The profile of the activator $u$ is plotted in grayscale. Black coding corresponds to $u=1$, and white to $u=0$. Parameters used are those of Fig. 1, plus $\varepsilon=0.01$.

than those of the deterministic dynamics. In order to model this situation, we add to $b$ a zero-mean Gaussian noise $\eta(x, t)$ with correlation

$$
\left\langle\eta(x, t) \eta\left(x^{\prime}, t^{\prime}\right)\right\rangle=2 C\left(x-x^{\prime}\right) \delta\left(t-t^{\prime}\right),
$$

which corresponds to a spatially extended stochastic process white in time and with a spatial correlation function $C(x)$. We note that this noise is multiplicatively coupled to the state of the system, since it leads in the activator Eq. (1a) to an additional random term of the form

$$
\phi(u)=\frac{1}{a \varepsilon} u(u-1) \eta(x, t) .
$$

The assumption of such a multiplicative noise is not unreasonable, given that the FitzHugh-Nagumo equations constitute a qualitative simplification of the well-known HodgkinHuxley model of electrical signalling in neurons, basically consisting in reducing the fast dynamics of the sodium conductance of the cell membrane $[2,19]$. In such a reduction process, additive fluctuations of this conductance would readily give rise to multiplicative noise terms in the activator equation. Our simplified choice of the noise term corresponds in fact to a fluctuating excitation threshold (since $b$ is the offset of the tilted $u$ nullcline in Fig. 1). This is the approach followed in the experiments on the photosensitive Belousov-Zhabotinsky reaction $[14,15]$, which can be modeled directly by the Barkley equations, with $b$ corresponding to the fluctuating illumination level [27]. We also note that the random term $\phi(u)$ vanishes identically for $u=0$ and 1 , so that the fluctuations do not perturb the system neither in the rest nor in the excited state. This is very different from an additive or other types of multiplicative noise [21], where the fluctuations actively perturb the system in some or all of the fixed points. In particular, the noise we consider here is not able to induce a decay from one of stable steady states towards the other.

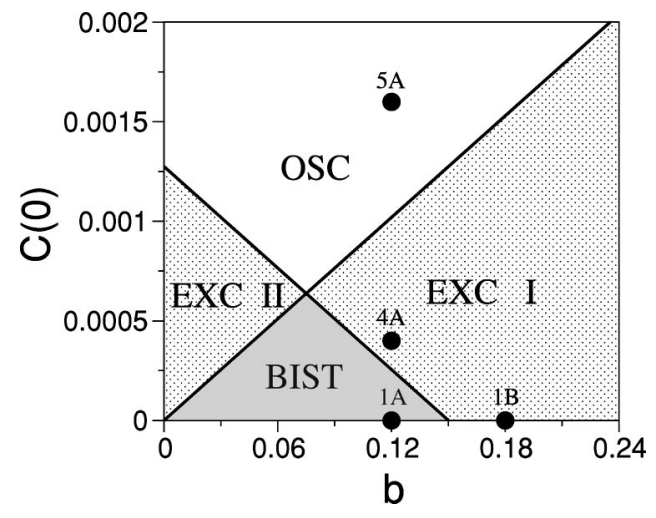

FIG. 3. Phase diagram of the Barkley model with external noise in the $C(0)-b$ plane. Other parameters are those of Fig. 2. The four labeled points denote four different regimes represented in the corresponding figures.

In order to establish the effect of the multiplicative noise $\eta(x, t)$ in the deterministic dynamics described in the previous section, we note that the random term (4) has a nonzero mean equal to [28]

$$
\langle\phi\rangle=\frac{1}{\varepsilon^{2} a^{2}} C(0)\langle u(u-1)(2 u-1)\rangle,
$$

where the brackets denote averaging over the probability distribution of the multiplicative noise, which has been interpreted in the Stratonovich sense. According to Eq. (5), the random term gives rise to a systematic nonzero contribution to the average dynamics of the system. This systematic contribution can be incorporated explicitly into the activator equation as the first-order term of a small-noise expansion [28], where the remaining stochastic contributions of the noise average out to zero. In that way, one can write down an effective equation for the activator dynamics, which happens to have exactly the same form as the original deterministic model, but with renormalized parameter values:

$$
\begin{gathered}
a^{\prime}=a-\frac{2 C(0)}{\varepsilon a}, \\
b^{\prime}=b-\frac{C(0)}{\varepsilon a}, \\
\varepsilon^{\prime}=\frac{\varepsilon}{1-2 C(0) / \varepsilon a^{2}},
\end{gathered}
$$

where the noise correlation at zero distance $C(0)$ measures the strength of the noise. We can thus see that even though the random fluctuations only affect directly the parameter $b$, they produce a renormalization of all three parameters of the activator dynamics, in the sense of decreasing the effective values of $a$ and $b$, and increasing that of $\varepsilon$. These renormalizations lead to important displacements of the boundary lines between the different dynamical regimes exhibited by this model, as shown in Fig. 3. This figure plots the phase diagram of the system in the plane formed by the parameters $b$ and $C(0)$, for constant values of $a=0.85$ and $\gamma=0.7$. Sev- 
(b)
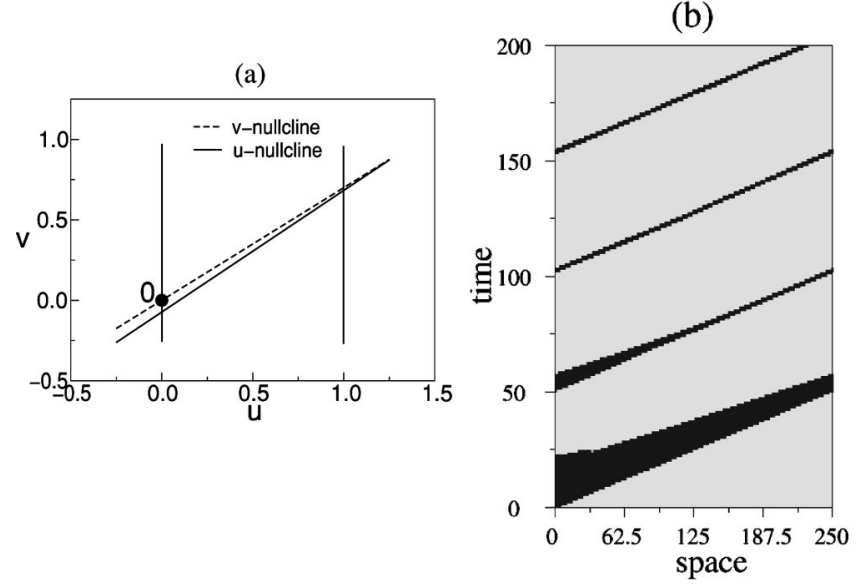

FIG. 4. Noise-induced excitability in the bistable regime of Fig. 2(a): (a) effective nullclines for $C(0)=4 \times 10^{-4}$; (b) corresponding spatiotemporal behavior numerically simulated for $\sigma^{2}=10^{-4}$ and $\Delta x=0.25$.

eral remarkable features in this phase diagram should be noted. First, the transition between the bistable and excitable regimes of Fig. 1 (labeled BIST and EXC I, respectively, in Fig. 3) is advanced as noise intensity increases. This leads to an enlargement of the region of excitable behavior, in such a way that a deterministically bistable medium can become excitable for large enough intensity of the multiplicative noise. An example of this noise-induced excitable dynamics is given in Fig. 4, which shows the behavior of the system in the deterministically bistable regime of Fig. 1(a), but with an amount of external noise corresponding to the point labeled 4 in the phase diagram of Fig. 3. The location of the effective nullclines in phase space is shown in Fig. 4(a), which clearly exhibits an excitable layout. Plot 4(b) displays the corresponding spatiotemporal dynamics, as simulated for an external noise taken to be white in the discrete space defined by the $1 \mathrm{D}$ lattice. In this case, $C(0)=\sigma^{2} / \Delta x$, where $\Delta x$ is the mesh size of the lattice. Following a local excitation in the left boundary of the medium, consisting only in a instantaneous suprathreshold increase of the activator concentration, the system self organizes after a certain transient into an excitable pulse. We stress again that this effect cannot be merely due to a noise-induced decay from the metastable state 1 [as labeled in Fig. 1(a)], since the random term (4) vanishes in that state. Hence, this behavior must correspond to a dynamical destabilization of state 1 due to the fluctuations, following a noise-induced transition denoted by the corresponding line in the phase diagram of Fig. 3. Extensive numerical simulations confirm the existence of such a clearcut transition, showing that below the transition line, the system develops fronts systematically, independent of the noise realization, whereas above it only pulses are found. In fact, beyond the transition point, pulses are the only stable structures of the system, and fronts cannot be obtained no matter the initial conditions considered.

A second feature to be noted in the phase diagram of Fig. 3 is the appearance of a second excitable regime, labeled EXC II in the figure, which is symmetric to that represented
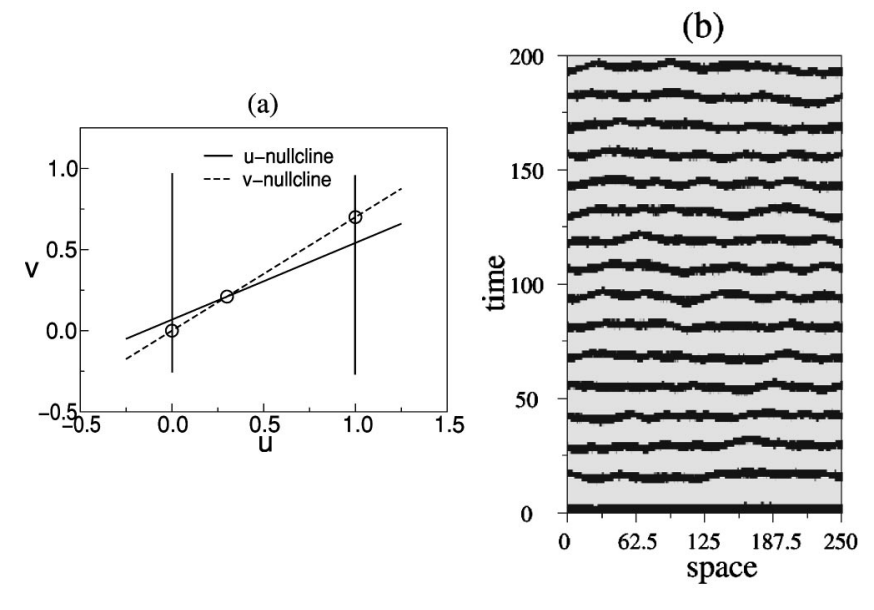

FIG. 5. Noise-induced oscillatory dynamics in the bistable regime of Fig. 2(a): (a) effective nullclines for $C(0)=1.6 \times 10^{-3}$; (b) corresponding spatiotemporal behavior for $\sigma^{2}=4 \times 10^{-4}$ and $\Delta x$ $=0.25$.

in Fig. 4(a). For this regime, the effective nullclines are such that the only remaining stable fixed point is state 1 , so that now the system allows for the propagation of inverted pulses, with the excited branch corresponding to $u \approx 0$, and the rest state to $u=1, v=\gamma$. This dynamical regime exists in the deterministic model for $b<0$, and the corresponding transition from the bistable regime is now retarded for increasing noise intensity, so that the excitable regime is again enlarged.

Finally, a more unexpected feature arises in the phase diagram of Fig. 3 as the intensity of the noise is further increased. Such an increase produces a reduction in the effective slope of the tilted $u$ nullcline (controlled by $a$ ), which finally leads to the situation portrayed in Fig. 5(a). In this case, all three fixed points are unstable and the system develops a limit cycle, which corresponds to a noise-induced oscillatory regime (labeled OSC in Fig. 3), analogous to the one recently observed experimentally in deterministically excitable media [15]. The related spatiotemporal behavior as computed from numerical simulations is represented in Fig. 5(b), where random initial conditions have been chosen. In this regime, pulses are spontaneously nucleated simultaneously throughout the system, which leads to a strongly synchronized firing of all lattice sites. Again, the transition between the excitable and oscillatory regime is clear-cut defined, as can be checked by extensive numerical simulations through the whole parameter space. We stress here that such an oscillatory dynamics does not exist in the deterministic system for any value of $b$, given the values of $a>\gamma$ chosen here. Furthermore, it can be seen that the dynamics shown in Figs. 5(b) shuts down to the rest state 0 as soon as the external noise is switched off.

\section{NOISE EFFECTS IN PULSE PROPAGATION}

The effective model derived in the previous section can be further verified by examining the effect of its renormalized parameters on the characteristics of the propagating pulses, such as their speed and width. The speed of an activator- 


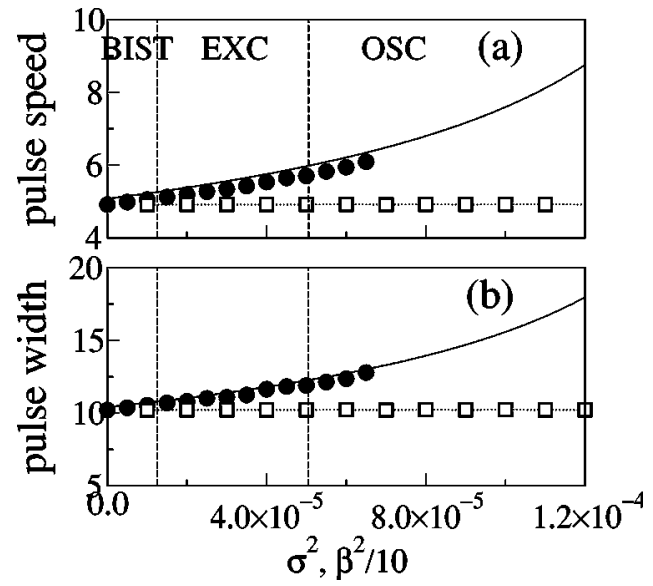

FIG. 6. Dependence of pulse speed (a) and width (b) on the external noise intensity. Parameters are those of Fig. 2(a). Solid lines represent the analytic results from the effective theory, and symbols numerical simulations in a lattice of 1000 sites with $\Delta x$ $=0.05$, with full circles corresponding to multiplicative noise of intensity $\sigma^{2}$, and empty squares to additive noise of intensity $\beta^{2}$.

inhibitor pulse can be easily evaluated in an approximate way by assuming a vanishingly small time-scale ratio $\varepsilon$. Within that approximation, the speed of the leading front of the pulse (the one going from $u=0$ to $u=1$ ), which corresponds in fact to the speed of the pulse itself, can be easily determined $[1,19]$. For the Barkley model, its value is

$$
c=\frac{1}{\sqrt{2 \varepsilon^{\prime}}}\left(1-2 \frac{b^{\prime}}{a^{\prime}}\right),
$$

where the renormalized parameters $a^{\prime}, b^{\prime}$, and $\varepsilon^{\prime}$ are given by Eqs. (6). This result predicts that the pulse speed will grow with increasing noise intensity, as shown by the solid line in Fig. 6(a), and in agreement with previous results on front propagation in one-component bistable media [29]. This analytical prediction can be verified by systematic numerical simulations, as shown by full circles in Fig. 6(a), which represents the time-averaged pulse speed in the steady state. The deterministic parameters are those of the bistable regime of Fig. 1(a), which undergoes two transitions as the multiplicative noise intensity increases. These two transitions, first towards the excitable regime and later towards the oscillatory one, are represented in Fig. 6 by vertical dashed lines. Pulses in the bistable regime are obtained only by special initial conditions [25]. In the oscillatory regime a pulse train is obtained, from which the speed of a single pulse can be measured.

The nontrivial influence of multiplicative noise in this system can be readily recognized by comparing the dependence of pulse speed on noise intensity with that corresponding to additive noise. To that end, we consider the effect of a simple additive noise, white in space and time with zero mean and intensity $\beta^{2}$, in the activator equation. The speed of the pulse in this case is represented as empty squares in Fig. 6(a). It can be seen that, although such a noise is also able to produce excitablelike pulses in the bistable regime (by means of a noise-induced decay mechanism) it does not affect the underlying dynamics of the system, since it does not vary the speed of propagation of the pulses (and does not produce the transitions indicated in the figure).

A second quantity that can be evaluated in a straightforward way from the renormalized theory is the pulse width $w$. This magnitude is equal to $w=c T$, where $T$ is the time that the system locally spends in the excited branch. Assuming again a vanishingly small $\varepsilon$, this quantity is simply $T$ $=\int_{0}^{u_{m}} d v / g(1, v)$, where $v_{m}$ is the maximum value reached by the inhibitor $v$, which can be calculated by imposing that the speeds of the leading and trailing fronts of the pulse are equal [19]. With this approach, $v_{m}$ can be seen to be given by $v_{m}=a^{\prime}-2 b^{\prime}$, which happens not to depend on the noise intensity, since the two contributions from $a^{\prime}$ and $b^{\prime}$ cancel each other. Thus, $T$ is not modified by the noise strength either, and the pulse width is given by $w=c \ln [\gamma /(\gamma+2 b$ $-a)$. Therefore, the width of the pulse increases with noise intensity as well, as shown by the solid line in Fig. 6(b), a prediction that is also verified by numerical simulations, represented by full circles in the same figure. Additive noise, on the other hand, always produces pulses of the same width.

\section{CONCLUSIONS}

We have derived in the previous sections an effective Barkley model that accounts for the influence of multiplicative noise in the activator dynamics of the system as the firstorder term of a small-noise expansion. The phase diagram of the resulting renormalized model predicts a general enhancement of the excitable behavior of the system for small-noise intensities, as well as the appearance of an oscillatory regime for larger-noise intensities, for which the system exhibits spontaneous nucleation of pulses that leads to synchronized firing. All these predictions have been verified by numerical simulations. The excitability enhancement leads to a destabilization of front propagation due to noise, and allows the propagation of pulses in situations where only front propagation could arise in the absence of noise. This effect can be understood as a mechanism for sustained signal propagation in spatially extended bistable systems. Even though we have considered here the case of continuous media, our results can also be applied to discrete arrays of locally coupled bistable elements. The nontrivial influence of random fluctuations is further verified by analyzing the dependence of the characteristics of propagating pulses, such as pulse speed and width, on the noise intensity. Our analysis shows that both the speed and width of the pulses grow with increasing noise strength, a fact that is verified by numerical simulations.

Experimental evidence shows bistable behavior in an increasing variety of neural systems, such as thalamocortical neurons [30] and mitral cells [31]. Our results demonstrate that signal propagation is possible even in such a bistable regime, and could thus provide an interpretation of the excitable behavior observed in noisy neural media, which has been usually associated so far only to locally excitable dynamics. 


\section{ACKNOWLEDGMENTS}

This work was supported by the DGES (Spain), under Project Nos. PB98-0935, BFM2000-0624, and BXX2000-
0638, and by the Sfb 555 (Germany). J.G.O. wishes to thank the Alexander von Humboldt-Stiftung for financial support. Computing resources from CEPBA (Spain) are also acknowledged.
[1] A. Mikhailov, Foundations of Synergetics I, 2nd ed. (Springer, Berlin, 1994).

[2] J. Keener and J. Snyder, Mathematical Physiology (Springer, New York, 1998).

[3] C. Fohlmeister, W. Gerstner, R. Ritz, and J. L. Van Hemmen, Neuron. Comp. 7, 905 (1995).

[4] P. Jung and G. Mayer-Kress, Phys. Rev. Lett. 74, 2130 (1995).

[5] D. R. Chialvo, G. A. Cecchi, and M. O. Magnasco, Phys. Rev. E 61, 5654 (2000).

[6] P. Jung and P. C. Galley, Ann. Phys. (Leipzig) 9, 697 (2000).

[7] E. Pálsson and E. C. Cox, Proc. Natl. Acad. Sci. U.S.A. 93, 1151 (1996).

[8] P. Jung, A. Cornell-Bell, K. S. Madden, and F. Moss, J. Neurophysiol. 79, 1098 (1998).

[9] H. Hempel, L. Schimansky-Geier, and J. García-Ojalvo, Phys. Rev. Lett. 82, 3713 (1999).

[10] C. Kurrer and K. Schulten, Phys. Rev. E 51, 6213 (1995).

[11] A. Neiman, L. Schimansky-Geier, A. Cornell-Bell, and F. Moss, Phys. Rev. Lett. 83, 4896 (1999).

[12] A. S. Pikovsky and J. Kurths, Phys. Rev. Lett. 78, 775 (1997).

[13] S. K. Han, T. G. Yim, D. E. Postnov, and O. V. Sosnovtseva, Phys. Rev. Lett. 83, 1771 (1999).

[14] S. Kádár, J. Wang, and K. Showalter, Nature (London) 391, 770 (1998).

[15] S. Alonso, I. Sendiña-Nadal, V. Pérez-Muñuzuri, J. M. Sancho, and F. Sagués, Phys. Rev. Lett. 87, 078302 (2001).

[16] M. Löcher, D. Cigna, and E. R. Hunt, Phys. Rev. Lett. 80, 5212 (1998).

[17] Y. Zhang, G. Hu, and L. Gammaitoni, Phys. Rev. E 58, 2952 (1998); J. F. Lindner, S. Chandramouli, A. R. Bulsara, M. Löcher, and W. L. Ditto, Phys. Rev. Lett. 81, 5048 (1998).

[18] J. García-Ojalvo, A. M. Lacasta, F. Sagués, and J. M. Sancho, Europhys. Lett. 50, 427 (2000).
[19] J. D. Murray, Mathematical Biology, 2nd ed. (Springer, Berlin, 1993).

[20] J. García-Ojalvo and L. Schimansky-Geier, Europhys. Lett. 47, 298 (1999).

[21] J. García-Ojalvo and L. Schimansky- Geier, J. Stat. Phys. 101, 473 (2000).

[22] D. Barkley, M. Kness, and L. S. Tuckerman, Phys. Rev. A 42, 2489 (1990).

[23] This transition has been investigated in a space-independent cubic-nullcline FitzHugh-Nagumo model by G. G. Izús, R. R. Deza, and H. S. Wio, Comput. Phys. Commun. 121, 406 (1999).

[24] Due to the absorbing boundaries at $u=0$ and $u=1$, only the fixed points in $0 \leqslant u \leqslant 1$ are accessible to the system for initial conditions within this region.

[25] Pulse generation in the bistable regime requires an initial condition in the form of a local activator excitation surrounded by inhibitor in only one side, which induces a symmetry break and produces a pulse propagation in the direction where the inhibitor is absent. These requirements are similar to those needed for the generation of isolated spirals in twodimensional excitable media.

[26] D. Barkley, Physica D 49, 61 (1991).

[27] J. Wang, S. Kádár, P. Jung, and K. Showalter, Phys. Rev. Lett. 82, 855 (1999).

[28] J. García-Ojalvo and J. M. Sancho, Noise in Spatially Extended Systems (Springer, New York, 1999).

[29] J. Armero, J. M. Sancho, J. Casademunt, A. M. Lacasta, L. Ramírez-Piscina, and F. Sagués, Phys. Rev. Lett. 76, 3045 (1996).

[30] S. W. Hughes, D. W. Cope, T. I. Tóth, S. R. Williams, and V. Crunelli, J. Physiol. (London), 517, 805 (1999).

[31] P. Heyward, M. Ennis, A. Keller, and M. T. Shipley, J. Neurosci. 21, 5311 (2001). 